\title{
Women Household Decision Making Power and Maternal Mortality
}

\author{
Yahaya Yakubu $^{1 *} \quad$ Norashidah Mohamed Nor $^{2} \quad$ Law Siong Hook $^{3} \quad$ Rusmawati Said $^{4}$ \\ ${ }^{1}$ Department of Economics, Bauchi State University Gadau, Bauchi State, Nigeria; fatyat2009@gmail.com \\ ${ }^{2}$ Faculty of Economics and Management, University Putra Malaysia; norashidah@upm.edu.my \\ ${ }^{3}$ Faculty of Economics and Management, University Putra Malaysia; lawsh@upm.edu.my \\ ${ }^{4}$ Faculty of Economics and Management, University Putra Malaysia; rusmawatisaid@gmail.com \\ "Correspondence: fatyat2009@gmail.com; +2348065428727
}

\begin{abstract}
High maternal mortality in the developing countries, particularly in Nigeria, poses serious challenge to achieving the maternal mortality target of the Sustainable Development Goals (SDGs) in the countries. Hence, there is need for multifaceted approach to curtailing the scourge. Women being the victims of maternal mortality, this study finds the effect of their household decision making power in reducing maternal mortality. The study used data from the 2013 Nigeria Health and Demographic Survey (NDHS) and logistic regression model to explore the relationship between women household decision making power and maternal mortality in Nigeria. The finding shows that women who decide and participate in household decision on own health, major purchases and visit to family and relatives were $35 \%(\mathrm{OR}=0.65,95 \%$ CI: $0.52,0.83), 27 \%(\mathrm{OR}=0.73,95 \% \mathrm{CI}: 0.57,0.92)$, and $37 \%$ (OR $=0.63,95 \%$ CI: 0.50, $0.80)$ less likely to experience maternal mortality, respectively, compared to those whose husbands alone decide. Women household decision making power is therefore instrumental to reducing maternal mortality. It is thus important for policy makers, particularly in Nigeria, to pay more attention to social and cultural factors that surround women household decision making ability for speedy reduction in maternal deaths.
\end{abstract}

Keywords: Women Household Decision Making; Maternal Mortality; Sustainable Development Goals; Developing countries; Nigeria 


\section{Introduction}

Reducing maternal mortality appears to be one of the major issues in world development agenda. The recently concluded Millennium Development Goals (MDGs) in 2015, and the current Sustainable Development Goals (SDGs) have reduction in Maternal Mortality Ratio (MMR) as one of their major targets. Maternal mortality is more prevalent in the developing countries. These countries are host to $99 \%$ of global maternal mortality [1,2]. In 2015, developing countries accounted for $99 \%(302,000)$ of the world total maternal mortality $(303,000)$. Nigeria as a developing country and one the sub-Saharan African countries had the highest number of maternal deaths in the world in 2015 [1] and ranked third among the 18 countries with very high MMR in the world as shown in figure 1. With such a high MMR, achieving the SDGs' target for MMR in Nigeria becomes a herculean task. Nigeria requires annual MMR reduction of $7.5 \%$ to achieve the SDGs' target of reducing MMR to at least 70 per 100,000 live birth by 2030. However, the current annual MMR reduction in Nigeria is 2.0\% [1]. Achieving the MMR target in the SDGs for Nigeria therefore requires the synergy of various factors to expedite the annual reduction in the MMR.

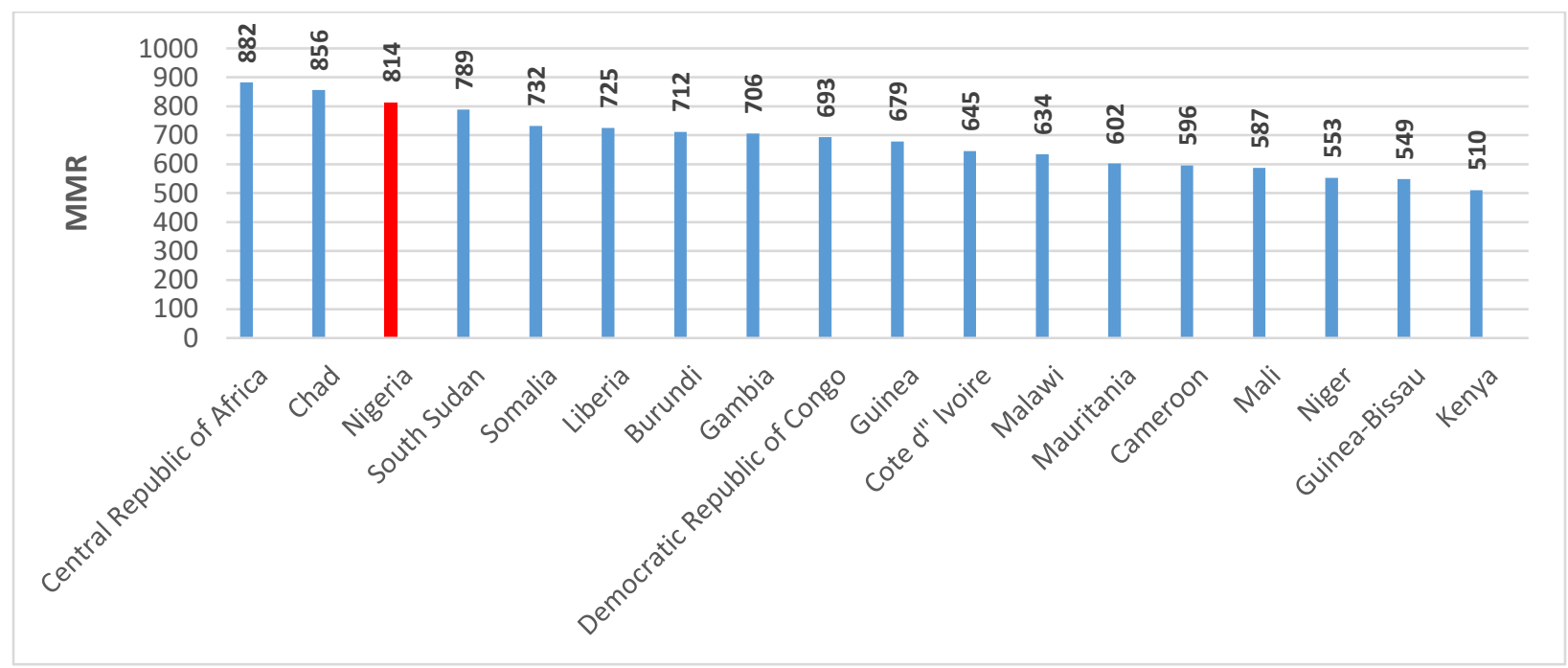

Figure 1: Countries with very high maternal mortality ratio (MMR) in the world in 2015

Source: [1] 
Nigeria is a country in the sub-Saharan African region. Being the most populous country in Africa and the $7^{\text {th }}$ in the world, Nigeria is estimated in 2017 to have population of 190,886000 people [3]. The population is almost equally divided across gender, 50.6\% and $49.4 \%$ of the population are males and females, respectively [4]. As obtains in the African continent at large, women in Nigeria suffer low status and autonomy vis-à-vis their male counterparts. Africa and Nigeria in particular are patriarchal settings where males are assumed to have the natural right of dominance over the females $[5,6]$. Such patriarchal belief imposes restrictions on the control and participation of women in matters that affect them and the society [7]. Hence, African and Nigerian women suffer low status and denial of household decision autonomy, particularly on their health, freedom of movement to seek for health and control of resources to purchase health services. Naturally, women are the victims of maternal mortality; denial of their participation in decisions that affect them could contribute to the high maternal mortality.

Women household decision making power is measured by the ability of women to take and participate in three major household's decision dimensions - decision on own health, decision on major household purchases and decision on visits to family and relatives [8$11]^{1}$. The ability of women to take and participate in any or all of these decisions making dimensions has been empirically revealed to improve maternal health care service utilization [10,12-16]. However, the direct effect of women household decision making in reducing maternal mortality has not been given attention in literature. This study extend this effect to the ultimate goal of maternal health care service utilization, which is reducing maternal deaths. Thus, this study investigate the effect of the three household decision making dimensions in reducing maternal mortality in Nigeria.

\footnotetext{
${ }^{1}$ Participation of women in household decision-making index, MEASURE Evaluation https://www.measureevaluation.org/prh/rh_indicators/gender/wgse/participation-of-women-in-household-decision
} 
Women household decision making plays essential role in women utilization of maternal healthcare services, which could reduce their risk of maternal death. Figure 2 depicts the processes through which women decision autonomy in the household could reduce the risk of maternal death. Women are considered as 'gold standard' with regard to maternal health knowledge and practices [17], and if their health is solely left in the hands of men, it gets less priority [16]. Mobility restriction and not having money deter women from accessing antenatal care and delivery in health facility, respectively $[16,18]$. Therefore, women's ability to participate in decision on their own health helps them prioritize their health and enables them to act promptly in seeking for health without necessarily being permitted by anyone. Taking part in decision on major household purchases avails them access to resources with which they can pay for timely healthcare such as delivery in health facility and postnatal care. Participating in decision on visit to family and relatives gives them liberty to move freely with no or limited restriction, and this could give them access to health such as antenatal care at the time of need. Hence, women household decision making ability can reduce the first, second and third delay in the three delays model for accessing maternal healthcare ${ }^{2}$, thereby facilitate maternal healthcare utilization in the form of accessing antenatal, delivering at health facility, getting the services of skilled birth attendant during birth and accessing postnatal care after birth. It is therefore presumable that women who participate in any or all the household decision making dimensions could have less risk of maternal mortality.

\footnotetext{
2 The three delays in the model are: delay in decision to seek healthcare, delay in reaching the healthcare and delay in receiving healthcare [34-36].
} 


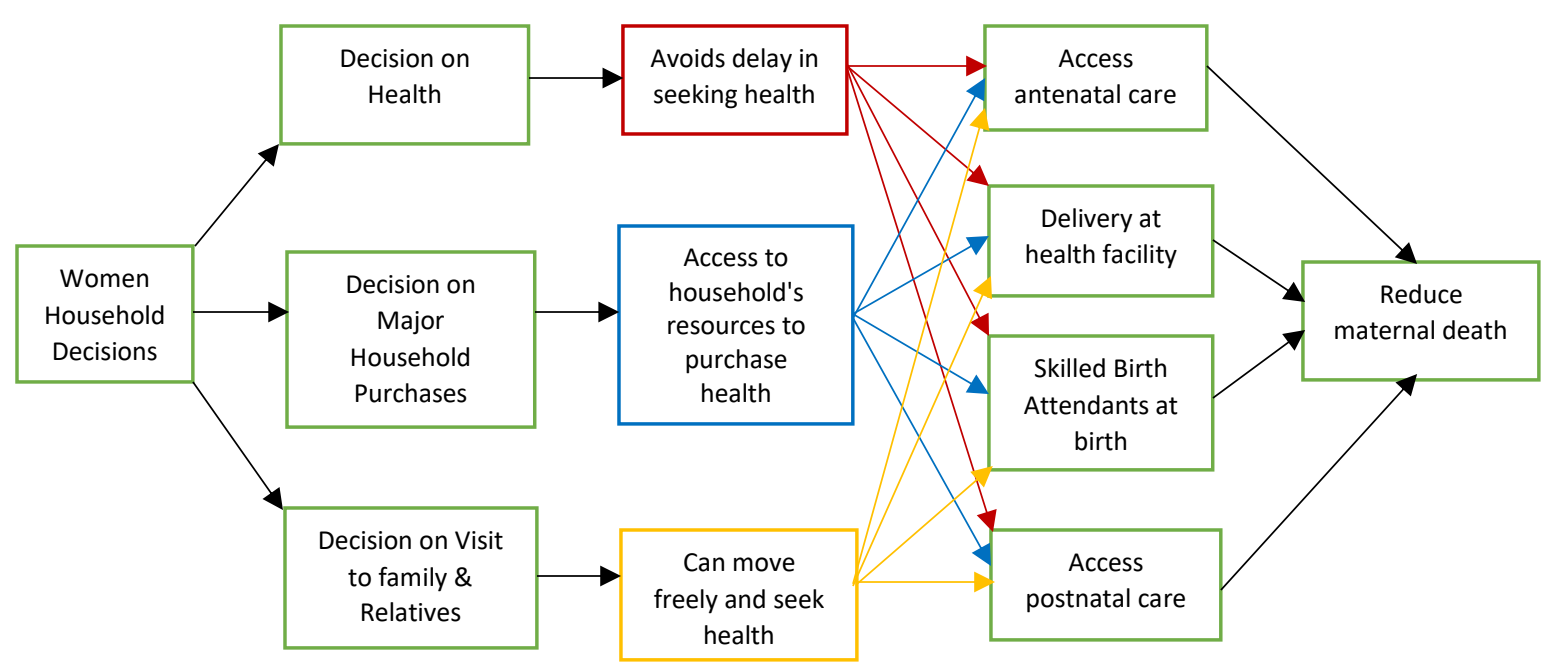

Figure 2: Conceptual framework for the relationship between women household decision making and maternal mortality

Source: Author

\section{Methods and Materials}

The study employs the national representative cross sectional data from the 2013 Nigeria Demographic and Health Survey (NDHS). The 2013 NDHS is the current and the fifth wave of the national survey, undertaken every five years. The NDHS provides current information on population health and socioeconomic situation in Nigeria, particularly in the area of fertility preference, fertility rate, use of contraceptives, women and children nutritional status, adult, maternal and childhood mortality, educational levels, wealth, and other population characteristics. In the 2013 NDHS, a sample of 39,902 women, aged 15-49, were identified as eligible for interview. Ninety eight percent (98\%) of the women were successfully interviewed [19]. However, only 3,302 responses were recorded for maternal mortality. Maternal mortality questions usually experience high non-response rate of $10 \%-40 \%$ in the Demographic and Health Survey (DHS) across many countries, and this could underestimate maternal mortality incidence [20]. Nevertheless, the DHS has importantly becomes source of data for maternal mortality, particularly in the developing world [21]. 
The outcome variable in the study is maternal mortality. Maternal mortality refers to the death of a woman associated with pregnancy, that occur within the period of pregnancy, child birth and postpartum period of 42 days after delivery or termination of the pregnancy [1]. In the 2013 NDHS, data on maternal mortality were collected through verbal autopsy, where surviving siblings were asked of the histories of their death sisters $[20,21]$. Series of questions were asked to ascertain whether a death was maternal death. Six basic responses were determined from the questions: she was never pregnant, her death not related to pregnancy, died after delivery, died since delivery, died six weeks after delivery, and died two months after delivery. The first two responses does not indicate maternal mortality while the remaining four responses revealed the occurrence of maternal mortality. In this study, the first two responses were coded "0" (not maternal mortality) and the remaining four responses were coded " 1 " (maternal mortality).

The main explanatory variable in the study is women household decision making power. Three indicators of household decision making were used - decision on health, major household purchases and visit to relatives and family. In the 2013 NDHS, women were asked on who take these decisions. The responses for each of the decisions were coded as "Respondent alone" = 1, "Respondent and Husband/partner" = 2, "Husband/partner alone" $^{\prime}=3$, "Someone else" $=4$, and "Others" $=5$. For convenience, this study recoded the responses as "Husband/partner alone" = 1, "Respondent (wife) decide and participate" $(1$ and 2$)=2$, and "Others" (4 and 5) =3. Other explanatory variables used as controlled variables were household and individual's characteristics: wife's education, husband/partner's education, parity, maternal age at birth, access to electricity, source of drinking water, sanitation, type of cooking fuels, frequency of reading newspaper and magazines, frequency of listening to radio, frequency of watching television, wealth, ethnicity and region. Since the victims of maternal mortality were 'non-existence' at the time of the survey, using the familial assumption, the household and individual 
characteristics of the surviving siblings were assumed to be that of the sisters [22,23]. The familial assumption or sisterhood assumption considers the socioeconomic characteristics to be the same for sisters of the same household.

The outcome (dependent) variable of the study is a dichotomous variable, which takes the value of either 1 or $0-1$ for maternal mortality and 0 for no maternal mortality.

$$
\text { Maternal Mortality }{ }_{i}=\left\{\begin{array}{l}
1-\text { if there is maternal mortality } \\
0-\text { if otherwise }
\end{array}\right.
$$

One of the suitable methods for analyzing such data is the logistic regression model [2426]. Though there are other methods that are used in analyzing data with dichotomous dependent variable, logistic regression has become the commonly used because its function is designed to produce probability range between 0 and 1 , which is the range of the dependent variable values [26]. The model for estimation establishes the logit functional relationship between the dependent variable, which is maternal mortality, and the covariates. Equation 1 modelled logistic function of the occurrence of maternal mortality and women household decision making.

$\operatorname{logit}\left[p\left(M M_{i}=1 / X_{i}\right)=\alpha+\beta_{1} W D H_{i}+\beta_{2} W D P_{i}+\beta_{3} W D V_{i}+\beta_{4} C V_{i}+\varepsilon_{i}\right.$

Where,

$X=\quad$ Vector of covariates of maternal mortality

$\alpha=$ Constant

$\varepsilon=\quad$ Error term

$i=1,2,3, \ldots \ldots \ldots \ldots$ number of observations

$\beta_{1}, \beta_{2}, \beta_{3}, \beta_{4}=$ Corresponding coefficients (odds ratios) of the explanatory variables

$M M=$ Maternal Mortality

$W D H=$ Women Household Decision on Health

$W D P=$ Women Household Decision on Major Purchases

$W D V=$ Women Household Decision on Visit to Relatives and Family

$C V=$ Control Variables 


\section{Results}

Table 1 presents the summary statistics of the women's household decision making indices, other socioeconomic variables and maternal mortality. In the household decision on women's health, $55.78 \%$ of the women had their health exclusively decided by their husbands, $43.90 \%$ either decided or participated in the decision, while $0.32 \%$ had their health decided by others. Maternal mortality was higher among women whose health was decided by their husbands alone than among women who decided and participated in their health decision (23.69\% versus $10.07 \%$ ). Similarly, $56.57 \%$ of the women had their husbands deciding alone in decision on household major purchases while $43.90 \%$ of the women either decided or participated in the decision. More than one-third of women whose husbands exclusively decided on this decision experienced maternal death while only about one-fourth of women who decided and participated in the decision experienced maternal death. Contrarily, in the decision on visit to relatives and family, $53.87 \%$ of the women decided and participated while $45.95 \%$ had their husbands decided alone and $0.18 \%$ decided by others. Though percentage of women who decided and participated in this decision was higher than those whose husbands decided alone, percentage of maternal mortality was however higher among women whose husbands decided alone (20.49\% versus $13.29 \%)$.

Table 1: Summary statistics of the explanatory variables and maternal mortality

\begin{tabular}{llcccc}
\hline Variable & Category & $\mathbf{N}$ & \multicolumn{3}{c}{ Maternal Mortality (MM) (\%) } \\
& & & MM = 0 & MM = 1 & Total \\
\hline Decision on own health & & $\mathbf{2 6 7 2}$ & & & \\
& Husband alone decides & & 32.09 & 23.69 & 55.78 \\
& Wife decides and participate & & 33.83 & 10.07 & 43.90 \\
& Others decides & & 0.23 & 0.09 & 0.32 \\
& Total & & $\mathbf{6 6 . 1 5}$ & $\mathbf{3 3 . 8 5}$ & $\mathbf{1 0 0 . 0 0}$ \\
& & $\mathbf{2 6 7 0}$ & & & \\
Decision on major purchases & Husband alone decides & & 32.90 & 23.67 & 56.57 \\
& Wife decides and participate & & 32.73 & 10.10 & 42.83 \\
& Others decides & & .50 & .10 & .60 \\
& Total & & $\mathbf{6 6 . 1 3}$ & $\mathbf{3 3 . 8 7}$ & $\mathbf{1 0 0 . 0 0}$
\end{tabular}


Decision on visit to relatives \&

family

Wife's education

Husband/Partner's education

Maternal age at marriage

Parity

Source of drinking water

Sanitation

Type of cooking fuels

Access to electricity

Type of cooking fuels
$\begin{aligned} & \text { Frequency of reading newspaper \& } \\ & \text { magazine }\end{aligned}$

magazine

Frequency of listening to radio

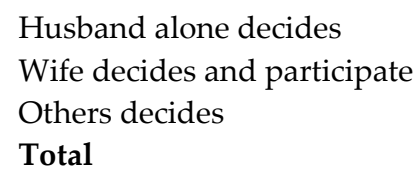

No education

Primary

Secondary

Higher

Total

No education

Primary

Secondary

Higher

Total

Nulliparous
$1-5$
$6-10$
$>10$
Total

$10-19$

$20-29$

$\geq 30$

Total

Access

No access

Total

Improved sources

Unimproved sources

Total

Improved

Unimproved

Total

Non-solid fuels
Solid fuels
Total

Not at all

Less than once in a week

Once in a week

Total

Not at all

\begin{tabular}{|c|c|c|}
\hline 25.46 & 20.49 & 45.95 \\
\hline 40.58 & 13.29 & 53.87 \\
\hline .12 & .06 & .18 \\
\hline 66.16 & 33.84 & 100.00 \\
\hline 20.69 & 18.11 & 38.80 \\
\hline 17.13 & 5.97 & 23.10 \\
\hline 22.46 & 6.89 & 29.35 \\
\hline 6.86 & 1.89 & 8.75 \\
\hline 67.14 & 32.86 & 100.00 \\
\hline 18.92 & 16.16 & 35.08 \\
\hline 16.06 & 5.90 & 21.96 \\
\hline 21.18 & 7.52 & 28.70 \\
\hline 10.56 & 3.70 & 14.26 \\
\hline 66.72 & 33.28 & 100.00 \\
\hline 30.87 & 8.37 & 39.24 \\
\hline 30.56 & 21.52 & 52.08 \\
\hline 5.14 & 3.24 & 8.38 \\
\hline .08 & .22 & .30 \\
\hline 66.65 & 33.35 & 100.00 \\
\hline 43.97 & 25.66 & 69.63 \\
\hline 21.23 & 6.971 & 28.20 \\
\hline 1.41 & .76 & 2.17 \\
\hline 66.61 & 33.39 & 100.00 \\
\hline 38.03 & 14.56 & 52.59 \\
\hline 28.99 & 18.42 & 47.41 \\
\hline 67.02 & 32.98 & 100.00 \\
\hline 39.72 & 19.07 & 58.79 \\
\hline 27.27 & 13.94 & 41.21 \\
\hline 66.99 & 33.01 & 100.00 \\
\hline 38.03 & 16.94 & 54.97 \\
\hline 28.97 & 16.06 & 45.03 \\
\hline 67.00 & 33.00 & 100.00 \\
\hline 1.52 & .24 & 1.76 \\
\hline 65.51 & 32.73 & 98.24 \\
\hline 67.03 & 32.97 & 100.00 \\
\hline 50.97 & 28.03 & 79.00 \\
\hline
\end{tabular}

3302

\begin{tabular}{|c|c|c|}
\hline 25.46 & 20.49 & 45.95 \\
\hline 40.58 & 13.29 & 53.87 \\
\hline .12 & .06 & .18 \\
\hline 66.16 & 33.84 & 100.00 \\
\hline 20.69 & 18.11 & 38.80 \\
\hline 17.13 & 5.97 & 23.10 \\
\hline 22.46 & 6.89 & 29.35 \\
\hline 6.86 & 1.89 & 8.75 \\
\hline 67.14 & 32.86 & 100.00 \\
\hline 18.92 & 16.16 & 35.08 \\
\hline 16.06 & 5.90 & 21.96 \\
\hline 21.18 & 7.52 & 28.70 \\
\hline 10.56 & 3.70 & 14.26 \\
\hline 66.72 & 33.28 & 100.00 \\
\hline 30.87 & 8.37 & 39.24 \\
\hline 30.56 & 21.52 & 52.08 \\
\hline 5.14 & 3.24 & 8.38 \\
\hline .08 & .22 & .30 \\
\hline 66.65 & 33.35 & 100.00 \\
\hline 43.97 & 25.66 & 69.63 \\
\hline 21.23 & 6.971 & 28.20 \\
\hline 1.41 & .76 & 2.17 \\
\hline 66.61 & 33.39 & 100.00 \\
\hline 38.03 & 14.56 & 52.59 \\
\hline 28.99 & 18.42 & 47.41 \\
\hline 67.02 & 32.98 & 100.00 \\
\hline 39.72 & 19.07 & 58.79 \\
\hline 27.27 & 13.94 & 41.21 \\
\hline 66.99 & 33.01 & 100.00 \\
\hline 38.03 & 16.94 & 54.97 \\
\hline 28.97 & 16.06 & 45.03 \\
\hline 67.00 & 33.00 & 100.00 \\
\hline 1.52 & .24 & 1.76 \\
\hline 65.51 & 32.73 & 98.24 \\
\hline 67.03 & 32.97 & 100.00 \\
\hline 50.97 & 28.03 & 79.00 \\
\hline
\end{tabular}

2914

\begin{tabular}{|c|c|c|}
\hline 25.46 & 20.49 & 45.95 \\
\hline 40.58 & 13.29 & 53.87 \\
\hline .12 & .06 & .18 \\
\hline 66.16 & 33.84 & 100.00 \\
\hline 20.69 & 18.11 & 38.80 \\
\hline 17.13 & 5.97 & 23.10 \\
\hline 22.46 & 6.89 & 29.35 \\
\hline 6.86 & 1.89 & 8.75 \\
\hline 67.14 & 32.86 & 100.00 \\
\hline 18.92 & 16.16 & 35.08 \\
\hline 16.06 & 5.90 & 21.96 \\
\hline 21.18 & 7.52 & 28.70 \\
\hline 10.56 & 3.70 & 14.26 \\
\hline 66.72 & 33.28 & 100.00 \\
\hline 30.87 & 8.37 & 39.24 \\
\hline 30.56 & 21.52 & 52.08 \\
\hline 5.14 & 3.24 & 8.38 \\
\hline .08 & .22 & .30 \\
\hline 66.65 & 33.35 & 100.00 \\
\hline 43.97 & 25.66 & 69.63 \\
\hline 21.23 & 6.971 & 28.20 \\
\hline 1.41 & .76 & 2.17 \\
\hline 66.61 & 33.39 & 100.00 \\
\hline 38.03 & 14.56 & 52.59 \\
\hline 28.99 & 18.42 & 47.41 \\
\hline 67.02 & 32.98 & 100.00 \\
\hline 39.72 & 19.07 & 58.79 \\
\hline 27.27 & 13.94 & 41.21 \\
\hline 66.99 & 33.01 & 100.00 \\
\hline 38.03 & 16.94 & 54.97 \\
\hline 28.97 & 16.06 & 45.03 \\
\hline 67.00 & 33.00 & 100.00 \\
\hline 1.52 & .24 & 1.76 \\
\hline 65.51 & 32.73 & 98.24 \\
\hline 67.03 & 32.97 & 100.00 \\
\hline 50.97 & 28.03 & 79.00 \\
\hline
\end{tabular}

3244

\begin{tabular}{|c|c|c|}
\hline 25.46 & 20.49 & 45.95 \\
\hline 40.58 & 13.29 & 53.87 \\
\hline .12 & .06 & .18 \\
\hline 66.16 & 33.84 & 100.00 \\
\hline 20.69 & 18.11 & 38.80 \\
\hline 17.13 & 5.97 & 23.10 \\
\hline 22.46 & 6.89 & 29.35 \\
\hline 6.86 & 1.89 & 8.75 \\
\hline 67.14 & 32.86 & 100.00 \\
\hline 18.92 & 16.16 & 35.08 \\
\hline 16.06 & 5.90 & 21.96 \\
\hline 21.18 & 7.52 & 28.70 \\
\hline 10.56 & 3.70 & 14.26 \\
\hline 66.72 & 33.28 & 100.00 \\
\hline 30.87 & 8.37 & 39.24 \\
\hline 30.56 & 21.52 & 52.08 \\
\hline 5.14 & 3.24 & 8.38 \\
\hline .08 & .22 & .30 \\
\hline 66.65 & 33.35 & 100.00 \\
\hline 43.97 & 25.66 & 69.63 \\
\hline 21.23 & 6.971 & 28.20 \\
\hline 1.41 & .76 & 2.17 \\
\hline 66.61 & 33.39 & 100.00 \\
\hline 38.03 & 14.56 & 52.59 \\
\hline 28.99 & 18.42 & 47.41 \\
\hline 67.02 & 32.98 & 100.00 \\
\hline 39.72 & 19.07 & 58.79 \\
\hline 27.27 & 13.94 & 41.21 \\
\hline 66.99 & 33.01 & 100.00 \\
\hline 38.03 & 16.94 & 54.97 \\
\hline 28.97 & 16.06 & 45.03 \\
\hline 67.00 & 33.00 & 100.00 \\
\hline 1.52 & .24 & 1.76 \\
\hline 65.51 & 32.73 & 98.24 \\
\hline 67.03 & 32.97 & 100.00 \\
\hline 50.97 & 28.03 & 79.00 \\
\hline
\end{tabular}

2936

\begin{tabular}{|c|c|c|}
\hline 25.46 & 20.49 & 45.95 \\
\hline 40.58 & 13.29 & 53.87 \\
\hline .12 & .06 & .18 \\
\hline 66.16 & 33.84 & 100.00 \\
\hline 20.69 & 18.11 & 38.80 \\
\hline 17.13 & 5.97 & 23.10 \\
\hline 22.46 & 6.89 & 29.35 \\
\hline 6.86 & 1.89 & 8.75 \\
\hline 67.14 & 32.86 & 100.00 \\
\hline 18.92 & 16.16 & 35.08 \\
\hline 16.06 & 5.90 & 21.96 \\
\hline 21.18 & 7.52 & 28.70 \\
\hline 10.56 & 3.70 & 14.26 \\
\hline 66.72 & 33.28 & 100.00 \\
\hline 30.87 & 8.37 & 39.24 \\
\hline 30.56 & 21.52 & 52.08 \\
\hline 5.14 & 3.24 & 8.38 \\
\hline .08 & .22 & .30 \\
\hline 66.65 & 33.35 & 100.00 \\
\hline 43.97 & 25.66 & 69.63 \\
\hline 21.23 & 6.971 & 28.20 \\
\hline 1.41 & .76 & 2.17 \\
\hline 66.61 & 33.39 & 100.00 \\
\hline 38.03 & 14.56 & 52.59 \\
\hline 28.99 & 18.42 & 47.41 \\
\hline 67.02 & 32.98 & 100.00 \\
\hline 39.72 & 19.07 & 58.79 \\
\hline 27.27 & 13.94 & 41.21 \\
\hline 66.99 & 33.01 & 100.00 \\
\hline 38.03 & 16.94 & 54.97 \\
\hline 28.97 & 16.06 & 45.03 \\
\hline 67.00 & 33.00 & 100.00 \\
\hline 1.52 & .24 & 1.76 \\
\hline 65.51 & 32.73 & 98.24 \\
\hline 67.03 & 32.97 & 100.00 \\
\hline 50.97 & 28.03 & 79.00 \\
\hline
\end{tabular}

3267

$38.03 \quad 14.56 \quad 52.59$

$28.99 \quad 18.42 \quad 47.41$

$\begin{array}{lll}67.02 & 32.98 & 100.00\end{array}$

3262

\begin{tabular}{|c|c|c|}
\hline 25.46 & 20.49 & 45.95 \\
\hline 40.58 & 13.29 & 53.87 \\
\hline .12 & .06 & .18 \\
\hline 66.16 & 33.84 & 100.00 \\
\hline 20.69 & 18.11 & 38.80 \\
\hline 17.13 & 5.97 & 23.10 \\
\hline 22.46 & 6.89 & 29.35 \\
\hline 6.86 & 1.89 & 8.75 \\
\hline 67.14 & 32.86 & 100.00 \\
\hline 18.92 & 16.16 & 35.08 \\
\hline 16.06 & 5.90 & 21.96 \\
\hline 21.18 & 7.52 & 28.70 \\
\hline 10.56 & 3.70 & 14.26 \\
\hline 66.72 & 33.28 & 100.00 \\
\hline 30.87 & 8.37 & 39.24 \\
\hline 30.56 & 21.52 & 52.08 \\
\hline 5.14 & 3.24 & 8.38 \\
\hline .08 & .22 & .30 \\
\hline 66.65 & 33.35 & 100.00 \\
\hline 43.97 & 25.66 & 69.63 \\
\hline 21.23 & 6.971 & 28.20 \\
\hline 1.41 & .76 & 2.17 \\
\hline 66.61 & 33.39 & 100.00 \\
\hline 38.03 & 14.56 & 52.59 \\
\hline 28.99 & 18.42 & 47.41 \\
\hline 67.02 & 32.98 & 100.00 \\
\hline 39.72 & 19.07 & 58.79 \\
\hline 27.27 & 13.94 & 41.21 \\
\hline 66.99 & 33.01 & 100.00 \\
\hline 38.03 & 16.94 & 54.97 \\
\hline 28.97 & 16.06 & 45.03 \\
\hline 67.00 & 33.00 & 100.00 \\
\hline 1.52 & .24 & 1.76 \\
\hline 65.51 & 32.73 & 98.24 \\
\hline 67.03 & 32.97 & 100.00 \\
\hline 50.97 & 28.03 & 79.00 \\
\hline
\end{tabular}

3267

\begin{tabular}{|c|c|c|}
\hline 25.46 & 20.49 & 45.95 \\
\hline 40.58 & 13.29 & 53.87 \\
\hline .12 & .06 & .18 \\
\hline 66.16 & 33.84 & 100.00 \\
\hline 20.69 & 18.11 & 38.80 \\
\hline 17.13 & 5.97 & 23.10 \\
\hline 22.46 & 6.89 & 29.35 \\
\hline 6.86 & 1.89 & 8.75 \\
\hline 67.14 & 32.86 & 100.00 \\
\hline 18.92 & 16.16 & 35.08 \\
\hline 16.06 & 5.90 & 21.96 \\
\hline 21.18 & 7.52 & 28.70 \\
\hline 10.56 & 3.70 & 14.26 \\
\hline 66.72 & 33.28 & 100.00 \\
\hline 30.87 & 8.37 & 39.24 \\
\hline 30.56 & 21.52 & 52.08 \\
\hline 5.14 & 3.24 & 8.38 \\
\hline .08 & .22 & .30 \\
\hline 66.65 & 33.35 & 100.00 \\
\hline 43.97 & 25.66 & 69.63 \\
\hline 21.23 & 6.971 & 28.20 \\
\hline 1.41 & .76 & 2.17 \\
\hline 66.61 & 33.39 & 100.00 \\
\hline 38.03 & 14.56 & 52.59 \\
\hline 28.99 & 18.42 & 47.41 \\
\hline 67.02 & 32.98 & 100.00 \\
\hline 39.72 & 19.07 & 58.79 \\
\hline 27.27 & 13.94 & 41.21 \\
\hline 66.99 & 33.01 & 100.00 \\
\hline 38.03 & 16.94 & 54.97 \\
\hline 28.97 & 16.06 & 45.03 \\
\hline 67.00 & 33.00 & 100.00 \\
\hline 1.52 & .24 & 1.76 \\
\hline 65.51 & 32.73 & 98.24 \\
\hline 67.03 & 32.97 & 100.00 \\
\hline 50.97 & 28.03 & 79.00 \\
\hline
\end{tabular}

3263

\begin{tabular}{|c|c|c|}
\hline 25.46 & 20.49 & 45.95 \\
\hline 40.58 & 13.29 & 53.87 \\
\hline .12 & .06 & .18 \\
\hline 66.16 & 33.84 & 100.00 \\
\hline 20.69 & 18.11 & 38.80 \\
\hline 17.13 & 5.97 & 23.10 \\
\hline 22.46 & 6.89 & 29.35 \\
\hline 6.86 & 1.89 & 8.75 \\
\hline 67.14 & 32.86 & 100.00 \\
\hline 18.92 & 16.16 & 35.08 \\
\hline 16.06 & 5.90 & 21.96 \\
\hline 21.18 & 7.52 & 28.70 \\
\hline 10.56 & 3.70 & 14.26 \\
\hline 66.72 & 33.28 & 100.00 \\
\hline 30.87 & 8.37 & 39.24 \\
\hline 30.56 & 21.52 & 52.08 \\
\hline 5.14 & 3.24 & 8.38 \\
\hline .08 & .22 & .30 \\
\hline 66.65 & 33.35 & 100.00 \\
\hline 43.97 & 25.66 & 69.63 \\
\hline 21.23 & 6.971 & 28.20 \\
\hline 1.41 & .76 & 2.17 \\
\hline 66.61 & 33.39 & 100.00 \\
\hline 38.03 & 14.56 & 52.59 \\
\hline 28.99 & 18.42 & 47.41 \\
\hline 67.02 & 32.98 & 100.00 \\
\hline 39.72 & 19.07 & 58.79 \\
\hline 27.27 & 13.94 & 41.21 \\
\hline 66.99 & 33.01 & 100.00 \\
\hline 38.03 & 16.94 & 54.97 \\
\hline 28.97 & 16.06 & 45.03 \\
\hline 67.00 & 33.00 & 100.00 \\
\hline 1.52 & .24 & 1.76 \\
\hline 65.51 & 32.73 & 98.24 \\
\hline 67.03 & 32.97 & 100.00 \\
\hline 50.97 & 28.03 & 79.00 \\
\hline
\end{tabular}

3288

$\begin{array}{lll}6.66 & 1.95 & 8.61\end{array}$

$\begin{array}{lll}67.29 & 32.71 & 100.00\end{array}$

3299

$\begin{array}{lll}20.93 & 12.15 & 33.08\end{array}$


Less than once in a week
Once in a week
Total

Frequency of watching television

Not at all
Less than once in a week
Once in a week
Total

Wealth

Region

$$
\begin{aligned}
& \text { Richest } \\
& \text { Rich } \\
& \text { Middle } \\
& \text { Poor } \\
& \text { Poorest } \\
& \text { Total }
\end{aligned}
$$

Ethnicity

Hausa/Fulani
Yoruba
Igbo
Others
Total

$\begin{array}{ccc}16.68 & 8.17 & 24.85 \\ 29.53 & 12.54 & 42.07 \\ \mathbf{6 7 . 1 4} & \mathbf{3 2 . 8 6} & \mathbf{1 0 0 . 0 0}\end{array}$

3293

$\begin{array}{ccc}26.80 & 18.48 & 45.28 \\ 13.02 & 5.25 & 18.27 \\ 27.39 & 9.06 & 36.45 \\ \mathbf{6 7 . 2 1} & \mathbf{3 2 . 7 9} & \mathbf{1 0 0 . 0 0}\end{array}$

3302

Southern region
Northern region
Total

$\begin{array}{ccc}16.80 & 4.32 & 21.12 \\ 15.02 & 5.83 & 20.85 \\ 14.21 & 6.47 & 20.68 \\ 12.07 & 7.43 & 19.50 \\ 9.04 & 8.81 & 17.85 \\ \mathbf{6 7 . 1 4} & \mathbf{3 2 . 8 6} & \mathbf{1 0 0 . 0 0} \\ & & \\ 15.12 & 15.57 & 30.69 \\ 10.60 & 2.75 & 13.35 \\ 11.26 & 3.24 & 14.50 \\ 30.18 & 11.28 & 41.46 \\ \mathbf{6 7 . 1 6} & \mathbf{3 2 . 8 4} & \mathbf{1 0 0 . 0 0} \\ & & \\ 33.23 & 8.18 & 41.41 \\ 33.92 & 24.67 & 58.59 \\ \mathbf{6 7 . 1 5} & \mathbf{3 2 . 8 5} & \mathbf{1 0 0 . 0 0}\end{array}$

Note: $\mathrm{MM}=0$ and $\mathrm{MM}=1$ refer to no maternal death and maternal death respectively

\subsection{Bivariate Analysis}

Table 2 presents the bivariate analysis of the association between women's household decision making, other explanatory variables, and maternal mortality. It shows the independent association between women's household decision making and maternal mortality. The result revealed that categories of women's household decision on health, major household purchases and visit to relatives and family were significantly different in women who did and did not experience maternal mortality at $\mathrm{p}<0.001$. With the significant independent association in the bivariate regression, women's household decision making is expected to have effect on maternal mortality even in the presence of other covariates. The controlled variables were also included in the bivariate regression in order to determine the variables to be included in the multivariate logistic regression. Only significant variables in the bivariate analysis are included in the multivariate analysis. The conservative significance level is $p<0.05$, however, this study set the 
threshold for significance in the bivariate regression to be $p<0.1$ in order to avoid the possibility of dropping any potential covariate from the multivariate analysis. Any variable not significant at $\mathrm{p}<0.1$ in table 2 was therefore dropped in the multivariate analysis.

Table 2: Bivariate logistic regression of the association between women household decision making, other explanatory variables, and maternal mortality

\begin{tabular}{|c|c|c|c|}
\hline Variable & $\mathbf{N}$ & Percentage (95\% C. I) & P-value \\
\hline Decision on own health & & & $<0.001$ \\
\hline Husband alone decides & 1,460 & $55.78(52.93,58.6)$ & \\
\hline Wife decides and participate & 1,203 & $43.9(41.08,46.76)$ & \\
\hline Others decides & 9 & $0.319(0.162,0.626)$ & \\
\hline Decision on major purchases & & & $<0.001$ \\
\hline Husband alone decides & 1,480 & $56.57(53.64,59.45)$ & \\
\hline Wife decides and participate & 1,173 & $42.83(39.96,45.74)$ & \\
\hline Others decides & 17 & $0.609(0.327,1.133)$ & \\
\hline Decision on visit to relatives \& family & & & $<0.001$ \\
\hline Husband alone decides & 1,192 & $45.95(42.88,49.06)$ & \\
\hline Wife decides and participate & 1,470 & $53.87(50.76,56.95)$ & \\
\hline Others decides & 7 & $0.18(0.075,0.431)$ & \\
\hline Wife's education & & & $<0.001$ \\
\hline No education & 1,213 & $38.8(35.94,41.74)$ & \\
\hline Primary & 785 & $23.1(20.98,25.37)$ & \\
\hline Secondary & 982 & $29.35(27.05,31.77)$ & \\
\hline Higher & 322 & $8.743(7.394,10.31)$ & \\
\hline Husband/Partner's education & & & $<0.001$ \\
\hline No education & 984 & $35.07(32.18,38.08)$ & \\
\hline Primary & 652 & $21.96(19.75,24.35)$ & \\
\hline Secondary & 820 & $28.7(26.34,31.17)$ & \\
\hline Higher & 458 & $14.27(12.41,16.35)$ & \\
\hline Parity & & & $<0.001$ \\
\hline Nulliparous & 1,295 & $39.24(37.22,41.31)$ & \\
\hline $1-5$ & 1,675 & $52.08(50.07,54.08)$ & \\
\hline $6-10$ & 268 & $8.373(7.262,9.636)$ & \\
\hline$>10$ & 9 & $.302(.139, .655)$ & \\
\hline Maternal age at marriage & & & $<0.001$ \\
\hline $10-19$ & 2,040 & $69.63(67.39,71.78)$ & \\
\hline $20-29$ & 827 & $28.2(26.13,30.36)$ & \\
\hline$\geq 30$ & 69 & $2.17(1.62,2.902)$ & \\
\hline Access to electricity & & & $<0.001$ \\
\hline Access & 1,678 & $52.59(49.09,56.08)$ & \\
\hline No access & 1,589 & $47.41(43.92,50.91)$ & \\
\hline Source of drinking water & & & 0.5541 \\
\hline Improved sources & 1,853 & $58.79(55.28,62.21)$ & \\
\hline Unimproved sources & 1,409 & $41.21(37.79,44.72)$ & \\
\hline Sanitation & & & 0.0330 \\
\hline Improved & 1,758 & $54.97(51.7,58.19)$ & \\
\hline Unimproved & 1,509 & $45.03(41.81,48.3)$ & \\
\hline
\end{tabular}




\begin{tabular}{|c|c|c|c|}
\hline Type of cooking fuels & & & 0.0089 \\
\hline Non-solid fuels & 61 & $1.761(1.137,2.718)$ & \\
\hline Solid fuels & 3,202 & $98.24(97.28,98.86)$ & \\
\hline $\begin{array}{l}\text { Frequency of reading newspaper and } \\
\text { magazines }\end{array}$ & & & $<0.001$ \\
\hline Not at all & 2,593 & $79.0(76.67,81.15)$ & \\
\hline Less than once in a week & 410 & $12.39(10.86,14.1)$ & \\
\hline Once in a week & 285 & $8.607(7.192,10.27)$ & \\
\hline Frequency of Listening to radio & & & 0.0210 \\
\hline Not at all & 1,159 & $33.08(30.7,35.55)$ & \\
\hline Less than once in a week & 821 & $24.85(22.77,27.04)$ & \\
\hline Once in a week & 1,319 & $42.07(39.54,44.65)$ & \\
\hline Frequency of watching television & & & $<0.001$ \\
\hline Not at all & 1,507 & $42.29(42.42,48,18)$ & \\
\hline Less than once in a week & 633 & $18.27(16.37,20.34)$ & \\
\hline Once in a week & 1,153 & $36.45(33.74,39.24)$ & \\
\hline Wealth & & & $<0.001$ \\
\hline Richest & 634 & $21.12(18.73,23.73)$ & \\
\hline Rich & 731 & $20.85(18.76,23.11)$ & \\
\hline Middle & 712 & $20.68(18.52,23.02)$ & \\
\hline Poor & 667 & $19.5(17.33,21.86)$ & \\
\hline Poorest & 558 & $17.85(15.46,20.51)$ & \\
\hline Ethnicity & & & $<0.001$ \\
\hline Hausa/Fulani & 906 & $30.69(27.74,33.82)$ & \\
\hline Yoruba & 392 & $13.35(11.37,15.61)$ & \\
\hline Igbo & 486 & $14.5(12.51,16.74)$ & \\
\hline Others & 1,514 & $41.46(38.44,44.55)$ & \\
\hline Region & & & $<0.001$ \\
\hline Southern part & 1,318 & $41.41(38.47,44.42)$ & \\
\hline Northern part & 1,984 & $58.59(55.58,61.53)$ & \\
\hline
\end{tabular}

\subsection{Multivariate Analysis}

Table 3 presents the results for the multivariate logistic regression for the association between women's household decision making power and maternal mortality. To ensure reliable coefficients in the multivariate regression, collinearity test among the potential covariates was conducted using the Pearson's $(\mathrm{r})$ Correlation test $(\mathrm{p}<0.05)$. Correlation was detected among the three indices of women household decision making, between wife's and husband's education, and between wife's education and frequency of watching television. Wife's education was thus dropped from the multivariate regression for husband's education despite its significance in the bivariate regression because husband's education plays more role in women's decision making autonomy [27]. To 
avoid having the correlated variables in the same model, three models were developed to capture all the three indices of women household decision making in the multivariate analysis.

Table 3: Multivariate logistic regression of the association between women household decision making power and maternal mortality

\begin{tabular}{|c|c|c|c|}
\hline Variable & $\begin{array}{c}\text { Model } 1 \\
\text { OR }(95 \% \text { CI) }\end{array}$ & $\begin{array}{c}\text { Model } 2 \\
\text { OR }(95 \% \mathrm{CI})\end{array}$ & $\begin{array}{c}\text { Model } 3 \\
\text { OR }(95 \% \text { CI) }\end{array}$ \\
\hline \multicolumn{4}{|l|}{ Decision on own health } \\
\hline Husband alone decides & 1.00 & & \\
\hline Wife decides and participate & $0.65(0.52,0.83)^{* * *}$ & & \\
\hline Others decides & $0.94(0.17,5.15)$ & & \\
\hline \multicolumn{4}{|l|}{ Decision on major purchases } \\
\hline Husband alone decides & & 1.00 & \\
\hline Wife decides and participate & & $0.73(0.57,0.92)^{* *}$ & \\
\hline Others decides & & $0.47(0.15,1.51)$ & \\
\hline \multicolumn{4}{|c|}{ Decision on visit to relatives \& family } \\
\hline Husband alone decides & & & 1.00 \\
\hline Wife decides and participate & & & $0.63(0.50,0.80)^{* * *}$ \\
\hline Others decides & & & $1.13(0.29,4.40)$ \\
\hline \multicolumn{4}{|l|}{ Husband/Partner's education } \\
\hline No education & 1.00 & 1.00 & 1.00 \\
\hline Primary & $0.78(0.56,1.08)$ & $0.77(0.55,1.06)$ & $0.78(0.56,1.08)$ \\
\hline Secondary & $0.95(0.65,1.39)$ & $0.94(0.64,1.37)$ & $0.95(0.65,1.39)$ \\
\hline Higher & $0.83(0.53,1.31)$ & $0.83(0.53,1.30)$ & $0.83(0.53,1.32)$ \\
\hline \multicolumn{4}{|c|}{$\begin{array}{l}\text { Frequency of reading newspaper and } \\
\text { magazines }\end{array}$} \\
\hline Not at all & 1.00 & 1.00 & 1.00 \\
\hline Less than once in a week & $0.92(0.62,1.36)$ & $0.90(0.61,1.33)$ & $0.90(0.61,1.34)$ \\
\hline Once in a week & $0.60(0.33,1.11)$ & $0.57(0.31,1.05)$ & $0.60(0.32,1.10)$ \\
\hline \multicolumn{4}{|l|}{ Frequency of Listening to radio } \\
\hline Not at all & 1.00 & 1.00 & 1.00 \\
\hline Less than once in a week & $1.12(0.82,1.53)$ & $1.10(0.81,1.50)$ & $1.12(0.82,1.52)$ \\
\hline Once in a week & $1.08(0.79,1.48)$ & $1.07(0.78,1.46)$ & $1.06(0.78,1.45)$ \\
\hline \multicolumn{4}{|l|}{ Frequency of watching television } \\
\hline Not at all & 1.00 & 1.00 & 1.00 \\
\hline Less than once in a week & $0.77(0.55,1.10)$ & $0.78(0.55,1.11)$ & $0.76(0.53,1.08)$ \\
\hline Once in a week & $0.89(0.62,1.28)$ & $0.92(0.64,1.33)$ & $0.90(0.62,1.31)$ \\
\hline \multicolumn{4}{|l|}{ Parity } \\
\hline No children & 1.00 & 1.00 & 1.00 \\
\hline $1-5$ & $2.43(1.93,3.05)^{* * *}$ & $2.40(1.91,3.02)^{* * *}$ & $2.42(1.93,3.04)^{* * *}$ \\
\hline $6-10$ & $1.66(1.15,2.39)^{* *}$ & $1.65(1.14,2.39)^{* *}$ & $1.65(1.14,2.38)^{* *}$ \\
\hline$>10$ & $7.69(1.90,31.07)^{* *}$ & $7.37(1.79,30.35)^{* *}$ & $7.18(1.72,29.92)^{* *}$ \\
\hline \multicolumn{4}{|l|}{ Maternal age at marriage } \\
\hline $20-29$ & 1.00 & 1.00 & 1.00 \\
\hline $10-19$ & $0.99(.76,1.31)$ & $0.99(0.75,1.31)$ & $0.98(0.74,1.28)$ \\
\hline$\geq 30$ & $1.85(0.96,3.56)$ & $1.89(0.98,3.65)$ & $1.83(0.95,3.53)$ \\
\hline \multicolumn{4}{|l|}{ Access to electricity } \\
\hline Access & 1.00 & 1.00 & 1.00 \\
\hline
\end{tabular}




\begin{tabular}{|c|c|c|c|}
\hline No access & $1.09(0.83,1.43)$ & $1.10(0.84,1.44)$ & $1.09(.83,1.42)$ \\
\hline \multicolumn{4}{|l|}{ Sanitation } \\
\hline Improved & 1.00 & 1.00 & 1.00 \\
\hline Unimproved & $1.02(0.80,1.31)$ & $1.03(0.80,1.32)$ & $1.05(0.82,1.34)$ \\
\hline \multicolumn{4}{|c|}{ Type of cooking fuels } \\
\hline Non-solid fuels & 1.00 & 1.00 & 1.00 \\
\hline Solid fuels & $1.46(0.47,4.54)$ & $1.58(0.49,5.08)$ & $1.56(0.49,4.94)$ \\
\hline \multicolumn{4}{|l|}{ Ethnicity } \\
\hline Others & 1.00 & 1.00 & 1.00 \\
\hline Hausa/Fulani & $1.77(1.27,2.47)^{* *}$ & $1.79(1.29,2.50)^{* *}$ & $1.81(1.30,2.51)^{* * *}$ \\
\hline Yoruba & $1.18(0.76,1.84)$ & $1.16(0.74,1.80)$ & $1.22(0.79,1.90)$ \\
\hline Igbo & $1.49(0.95,2.33)$ & $1.47(0.94,2.30)$ & $1.49(0.94,2.34)$ \\
\hline \multicolumn{4}{|l|}{ Region } \\
\hline Southern parts & 1.00 & 1.00 & 1.00 \\
\hline Northern parts & $1.98(1.37,2.88)^{* * *}$ & $2.01(1.39,2.90)^{* * *}$ & $1.90(1.32,2.75)^{* *}$ \\
\hline \multicolumn{4}{|c|}{ Model Specification Error Test } \\
\hline Linktest & $\begin{array}{c}\text { Coefficient } \\
(95 \% \text { CI })\end{array}$ & $\begin{array}{c}\text { Coefficient } \\
\text { (95\% CI) }\end{array}$ & $\begin{array}{c}\text { Coefficient } \\
(95 \% \mathrm{CI})\end{array}$ \\
\hline _hat & $0.86(0.63,1.09)^{* * *}$ & $0.87(0.64,1.10)^{* * *}$ & $0.86(0.65,1.08)^{* * *}$ \\
\hline hatsq & $-0.11(-0.25,0.04)$ & $-0.10(-0.26,-0.05)$ & $-0.11(-0.25,0.31)$ \\
\hline
\end{tabular}

Results of the multivariate regression in table 3 showed that, after controlling for household and individuals characteristics, women who had say in the decision that affects their health, either by deciding or participating in the decision, were $35 \%$ $(\mathrm{OR}=0.65 ; 95 \% \mathrm{CI}: 0.52,0.83, \mathrm{p}<0.001)$ less likely to experience maternal mortality compared to their counterparts who had this decision taken exclusively by their husbands/partners. Compared to women whose husband alone decided on major household purchases, women who decided and participated in this decision had 27\% ( $\mathrm{OR}=0.73 ; 95 \% \mathrm{CI}: 0.57,0.92, \mathrm{p}<0.01$ ) higher protection against maternal death. Similarly, the likelihood of maternal death among women who decided and participated in decision on visit to relatives and family was $37 \%(\mathrm{OR}=0.63$; 95\% CI: $0.50,0.80, \mathrm{p}<0.001)$ less compared to women whose only husbands decided. In addition to the significance of the main explanatory, other explanatory variables such as parity, being a Hausa/Fulani ethnic tribe and of northern region extraction were also significant and positively associated with maternal mortality in the multivariate analysis. All the three models were 
well specified as indicated by the post estimation test, the linktest, in table 3, the _hat was significance at $\mathrm{p}<0.001$ in all the models [28].

\section{Discussion}

The study gives empirical insight on the influence of women household decision making power in reducing maternal mortality. The findings revealed that indices of women household decision making, measured by decision on health, decision on household's purchase and decision on visit to family and relatives, were consistently significant and protective against maternal mortality. Previous studies mostly focused on association of women household decision making and the use of maternal health care services [911,15,16,29-32]. Best to the knowledge of this study, no prior empirical study on the association of women household decision making and maternal mortality, particularly in Nigeria, exists. This study therefore, extended the effect of women household decision making beyond maternal healthcare utilization to the ultimate health outcome, maternal mortality. From the multivariate result in table 3, women who decided and participated in household decision on health and visit to relatives and family were more protective against maternal mortality than those who decided and participated in decision on major household purchases (35\% and 37\%, respectively, compared to $27 \%$ protection). Though all the three decision indices are important to the prevention of maternal death, the implication of this difference is that, decision that is directly health and which affects mobility are more important and protective.

A woman is a gold standard for her health, the knowledge and practice, particularly as it relates to maternal health, is best known to her [17]. Hence, allowing her to take and partake in decision that affects her health gives her opportunity to take prompt action and makes her health a priority. Contrary to this, her health might suffer set-back. In a patriarchal setting where men exercise influential decision power, women suffer denial of permission to seek health during pregnancy which had led to maternal death as found 
in Costal Miskito, Honduras [33]. Similarly, women's mobility autonomy is also instrumental as it allows access to antenatal care, which gives room for preventive measures against any foreseen possible complication during delivery. If a woman decides and partakes in decision on visit to her relatives and family, it assumes that she has freedom of movement to access antenatal care. In the case of decision on major household purchases, though deciding and participating in it is equally important in the prevention against maternal death, it might not be as important as decision on health and visit to relatives and family. Taking and participating in this decision is assume to give the woman access to resources with which she can pay for maternal healthcare services or use for transportation to health facility. Maternal healthcare in public health facilities, particularly in developing countries are becoming free and primary healthcare centers are becoming closer to communities. Hence, decision on major household purchases might not be as important as decision on health and visit to relatives and family. With this finding, it is important that policy makers re-strategize their intervention on population health. Health policies and interventions should not only concentrate on health system, social factors such as the women's household decision making ability should also be given serious attention. To improve the women's household decision making power, factors that influence their decision making power in the household need to be identified for strategic policy action. This study thus recommend further study on factors that influence women household decision making power, particularly in Nigeria.

\section{Conclusion}

This study empirically examined the relationship between women household decision making power and maternal mortality. Three indices of household decision were used decision on health, household purchase and visit to relatives and family. The findings show that women household decision making power, in all its three dimensions, has significant influence in reducing maternal mortality in Nigeria. In the other words, 
maternal mortality can be reduced with improvement in women household decision making power. This finding is phenomenal, particularly with the SDGs in place and the challenges of developing countries such as Nigeria in achieving the maternal mortality target of the SDGs. Achieving the SDGs' target of maternal mortality requires expedient action through the synergy of different sectors and factors. Hence, in addition to other interventions, policy makers in Nigeria and other developing countries alike should leverage on women household decision making power to facilitate action towards achieving the SDGs' target.

Authors Contributions: Conceptualization, Yahaya Yakubu and Norashidah Mohamed Nor; Formal analysis, Yahaya Yakubu and Rusmawati Said; Methodology, Law Siong Hook; Writing - original draft, Yahaya Yakubu and Rusmawati Said; Writing - review \& editing, Yahaya Yakubu and Norashidah Mohamed Nor.

Funding: This research received no external funding

Acknowledgements: We wish to acknowledge and thank the United States Agency for International Development (USAID) for giving us access to the current NDHS data

Conflicts of Interest: The authors declare no conflict of interest.

\section{References}

1. World Health Organization. Trends in Maternal Mortality: 1990 to 2015 [Internet]. Estimates by WHO, UNICEF, UNFPA, World Bank Group and the United Nations Population Division. Geneva; 2015. Available from: http://www.who.int/reproductivehealth/publications/monitoring/maternalmortality-2015/en/

2. Yakubu Y, Mohamed Nor N, Abidin EZ. A systematic review of micro correlates of maternal mortality. Rev Environ Health. 2018;33(2):147-61.

3. United Nations. World Population Prospects: The 2017 Revision, Key Findings and Advance Tables (ESA/P/WP/248). New York; 2017. Available from: https://esa.un.org/unpd/wpp/Publications/

4. Central Intelligence Agency. Nigeria: people and society [Internet]. World Factbook. 2018. Available from: https://www.cia.gov/library/publications/the-world-factbook/geos/ni.html

5. Makama GA. Patriarchy and gender inequality in Nigeria: The way forward. Eur Sci J. 2013;9(17):115-44. Available from: https://eujournal.org/index.php/esj/article/view/1161

6. Sano Y, Sedziafa AP, Vercillo S, Antabe R, Luginaah I. Women's household decision-making autonomy and safer sex negotiation in Nigeria: An analysis of the Nigeria Demographic and Health 
Survey. AIDS Care - Psychol Socio-Medical Asp AIDS/HIV. 2018;30(2):240-5. Available from: https://doi.org/10.1080/09540121.2017.1363363

7. Jejeebhoy SJ, Sathar ZA. Women's autonomy in India and Pakistan: The influence of religion and region. Popul Dev Rev. 2001;27(4):687-712. Available from: https://doi.org/10.1111/j.17284457.2001.00687.x

8. Furuta M, Salway S. Women's position within the household as a determinant of maternal health care use in Nepal. Int Fam Plan Perspect. 2006;32(1):017-27. Available from: http://www.guttmacher.org/pubs/journals/3201706.pdf

9. Ghose B, Feng D, Tang S, Yaya S, He Z, Udenigwe O, et al. Women's decision-making autonomy and utilisation of maternal healthcare services: Results from the Bangladesh Demographic and Health Survey. BMJ Open. 2017;7:1-9.

10. Haider MR, Qureshi ZP, Khan MM. Effects of women's autonomy on maternal healthcare utilization in Bangladesh: Evidence from a national survey. Sex Reprod Healthc. 2017;14:40-7. Available from: https://doi.org/10.1016/j.srhc.2017.09.002

11. Hou X, Ma N. The effect of women's decision-making power on maternal health services uptake: Evidence from Pakistan. Health Policy Plan. 2013;28(2):176-84.

12. Pandey KK, Singh RD. Women's status, household structure and the utilization of maternal health services in Haryana, India. J Stat Appl Probab Lett. 2017;4(1):1-10.

13. Becker S, Fonseca-Becker F, Schenck-Yglesias C. Husbands' and wives' reports of women's decisionmaking power in Western Guatemala and their effects on preventive health behaviors. Soc Sci Med. 2006;62(9):2313-26.

14. Kamiya Y. Women's autonomy and reproductive health care utilisation: Empirical evidence from Tajikistan. Health Policy (New York). 2011;102(2-3):304-13.

15. Sado L, Spaho A, Hotchkiss DR. The influence of women's empowerment on maternal health care utilization: Evidence from Albania. Soc Sci Med. 2014;114:169-77. Available from: http://dx.doi.org/10.1016/j.socscimed.2014.05.047

16. Story WT, Burgard SA. Couples' reports of household decision-making and the utilization of maternal health services in Bangladesh. Soc Sci Med. 2012;75(12):2403-11. Available from: http://dx.doi.org/10.1016/j.socscimed.2012.09.017

17. Mullany BC. Spousal agreement on maternal health practices in Kathmandu, Nepal. J Biosoc Sci. 2010;42(5):689-93.

18. Matsuoka S, Aiga H, Rasmey LC, Rathavy T, Okitsu A. Perceived barriers to utilization of maternal health services in rural Cambodia. Health Policy (New York). 2010;95(2-3):255-63. Available from: http://dx.doi.org/10.1016/j.healthpol.2009.12.011

19. National Population Commission [Nigeria] and ICF International. Nigeria Demographic and Health Survey 2013. Abuja, Nigeria; 2014. Available from: https://dhsprogram.com/publications/publication-fr293-dhs-final-reports.cfm

20. Ahmed S, Li Q, Scrafford C, Pullum T. An Assesment of DHS Maternal Mortality Data and Estimates. DHS Methodological Reports No. 13. Rockville, Maryland, USA; 2014. Available from: https://www.dhsprogram.com/publications/publication-mr13-methodological-reports.cfm

21. Merdad L, Hill K, Graham W. Improving the Measurement of Maternal Mortality: The Sisterhood Method Revisited. PLoS One. 2013;8(4).

22. Graham WJ, Fitzmaurice AE, Bell JS, Cairns JA. The familial technique for linking maternal death with poverty. Lancet. 2004;363(9402):23-7.

23. Hussein J, Bell J, Iang MD, Mesko N, Amery J, Graham W. An appraisal of the maternal mortality decline in Nepal. PLoS One. 2011;6(5):1-10.

24. Cameron AC, Trivedi PK. Microeconometrics: Methods and applications. 1st editio. New York: Columbia University Press; 2005. 1-1058 p. Available from: www.cambridge.org /9780521848053 
25. Greene WWH. Econometric analysis . 7th editio. Yagan S, Battista D, D'Ambrosio A, Dent L, editors. New York: Pearson Education Limited; 2012. 1189 p. Available from: https://www.pearson.com/us/higher-education/program/Greene-Econometric-Analysis-7thEdition/PGM305107.html

26. Kleinbaum DG, Klein M. Logistic Regression: A Self-Learning Text. 3rd editio. Gail M, Krickeberg K, Samet JM, Tsiatis A, Wong W, editors. New York: Springler; 2010. 1-709 p.

27. Bhandari TR, Kutty VR, Sarma PS, Dangal G. Safe delivery care practices in western Nepal: Does women's autonomy influence the utilization of skilled care at birth? PLoS One. 2017;12(8):1-10.

28. Chen X, Ender PB, Mitchell M, Wells C. Logistic Regression with Stata. 2003 [cited 2017 Oct 22]. Available from: https://stats.idre.ucla.edu/stata/webbooks/logistic/

29. Ameyaw EK, Tanle A, Kissah-Korsah K, Amo-Adjei J. Women's health decision-making autonomy and skilled birth attendance in Ghana. Int J Reprod Med [Internet]. 2016;2016(6569514):1-9. Available from: https://www.hindawi.com/journals/ijrmed/2016/6569514/

30. Fawole OI, Adeoye IA. Women's status within the household as a determinant of maternal health care use in Nigeria. Afr Health Sci. 2015;15(1):217-225.

31. Mistry R, Galal O, Lu M. Women's autonomy and pregnancy care in rural India: A contextual analysis. Soc Sci Med. 2009;69(6):926-33. Available from: http://dx.doi.org/10.1016/j.socscimed.2009.07.008

32. Tiruneh FN, Chuang KY, Chuang YC. Women's autonomy and maternal healthcare service utilization in Ethiopia. BMC Health Serv Res. 2017;17(718):1-12.

33. Arps S. Threats to safe motherhood in Honduran Miskito communities: Local perceptions of factors that contribute to maternal mortality. Soc Sci Med [Internet]. 2009;69(4):579-86. Available from: http://dx.doi.org/10.1016/j.socscimed.2009.06.005

34. Bennouna C, Feldman B, Usman R, Adiputra R, Kusumaningrum S, Stark L. Using the Three Delays Model to examine civil registration barriers in Indonesia. PLoS One. 2016;11(12):1-15.

35. Thaddeus S, Maine D. Too far to walk: Maternal Mortality in context. Soc Sci Med [Internet]. 1994;38(8):1091-110. Available from: https://doi.org/10.1016/0277-9536(94)90226-7

36. Maternity Worldwide. The Three Delays Model and our Integrated Approach. Saving Lives in Childbirth. 2018. Available from: https://www.maternityworldwide.org/what-we-do/three-delaysmodel/ 